Classification

Physics Abstracts

$42.30 \mathrm{Q}-42.65 \mathrm{P}-42.80 \mathrm{~K}$

\title{
Optical data self-routing using address decoding
}

P. Koppa ( $\left(^{1}\right)$, P. Chavel ( $\left.{ }^{1}\right)$. J. L. Oudar $\left({ }^{2}\right)$, R. Kuszelewicz $\left({ }^{2}\right)$, J. Ph. Schnell $\left({ }^{3}\right)$ and J. P. Pocholle $\left(^{3}\right)$

(') Institut d'Optique Théorique et Appliquée, CNRS, B.P. 147, 91403 Orsay Cedex, France

(2) France Telecom CNET Paris-B, B.P. 107, 92225 Bagneux Cedex, France

$\left({ }^{3}\right)$ Thomson-CSF. LCR, Domaine de Corbeville, 91404 Orsay, France

(Received 15 April 1994, accepted 9 August 1994)

Résumé. - Nous présentons un autocommutateur optoélectronique pour signaux numériques organisés en paquets de bits. Le routage des paquets entrant dans le commutateur est déterminé par le décodage optique de l'adresse de destination présente dans l'en-tête de chaque paquet. Pour valider ce principe, un système démonstrateur de commutation d'une voie d'entrée vers 64 voies de sortie a été construit. L'expérience est basée sur l'association d'une matrice de $8 \times 8$ modulateurs électro-optiques et d'un composant bistable optique à structure de puits quantiques multiples en GaAs/GaAlAs. L'opération de décodage d'adresses a été montrée par nos premières expériences sur les voies du système prises une par une.

\begin{abstract}
We present an optoelectronic self-routing packet switch for digital data commutation applications. The routing of the input data is determined by the optical decoding of the destination address coded in the packet header preceding the data bits. As proof of the operating principle, a one input 64 output demonstration system was built. The experiment is based on the association of two $8 \times 8$ arrays of GaAs/GaAlAs multiple quantum well electro-optical modulators and optical bistable devices. We have demonstrated the optical address decoding in our first experiments by operating individually the channels of the set-up.
\end{abstract}

\section{Introduction.}

Optical technology is becoming more and more competitive for many applications in the fields of telecommunications, information processing and computing. The large bandwidth of optics and its immunity against electromagnetic perturbations are already fully applied in long distance communications via optical fibres. A good deal of recent research activity is focused on the area of free-space optical interconnections [1], which offers an additional gain through massive parallelism. Introducing optical switching devices into this scheme allows the realization of reconfigurable interconnects i.e. switching networks. Several optical/optoelectronic switching network implementations have been proposed using free-space beam propagation as interconnections and a great variety of active devices as switching node arrays [2-4]. In general, these networks are implemented with centralised control : an external 
processor receives requests for connections, computes data paths in the network and transmits control signals to switching nodes. With the growth of the number of inputs and outputs to the network, a serious communication bottleneck can occur between the control processor and the network. To avoid this difficulty, the use of distributed control seems to be a solution. In such a scheme, the setting of each switching node is determined by a local control unit, according to routing information arriving within the data stream. Generally, the input signal is organized in packet form, with the routing information placed in a packet header containing the destination address, possibly synchronisation, priority bits and/or other control signals. The treatment of the packet header can be performed by an electronic circuit integrated within the node. This circuit must include a logical unit to compute the node setting as a function of routing information, a memory to store the setting and an interface between memory and switch [5]. The alternative solution is to use optical distributed control. An example of this approach is the work of Buller et al. [6], who used optothermal nonlinear interference filters as 2 by 2 optical switches, each node being controlled by a header pulse. The optoelectronic architecture we propose implements switching by decoding a 6 bit long header address. Using this principle, the node complexity can be greatly reduced compared to electronic solutions, since the logical operations of the address decoding, the memorisation and the switching are performed by the same optical devices. To prove the validity of this principle a 1 input 64 output demonstration system was built, based on the association of an 8 by 8 pixel electrically addressed spatial light modulator and an optical bistable device. In the next section we give a brief description of system operation. Section 3 presents our first experimental results on the demonstration of address decoding in one channel of the system.

\section{Optical address decoding.}

The self-routing/address recognition operation is accomplished by the basic optical unit shown schematically in figure 1 . This unit includes two active devices : an electronically addressed 8 by 8 pixel spatial light modulator imaged on an 8 by 8 array of optical bistable devices, having the input-output characteristics shown in figure 2 .

Before the arrival of a signal packet into the routing unit, the bistable elements are initialised in their high reflectivity state and set to point $A$ of the hysteresis cycle using an $8 \times 8$ holding beam array. The input signal beam carries a pulse sequence organized in packet form, containing a packet header and the data to be transmitted. As shown in the first row of figure 3 , the packet header contains a synchronisation bit and the 6 bit destination address in temporal dual rail coding: i.e. a low-high sequence is emitted for bit 0 , high-low for bit 1 . The destination address is a binary number between 000000 and 111111 , corresponding to the

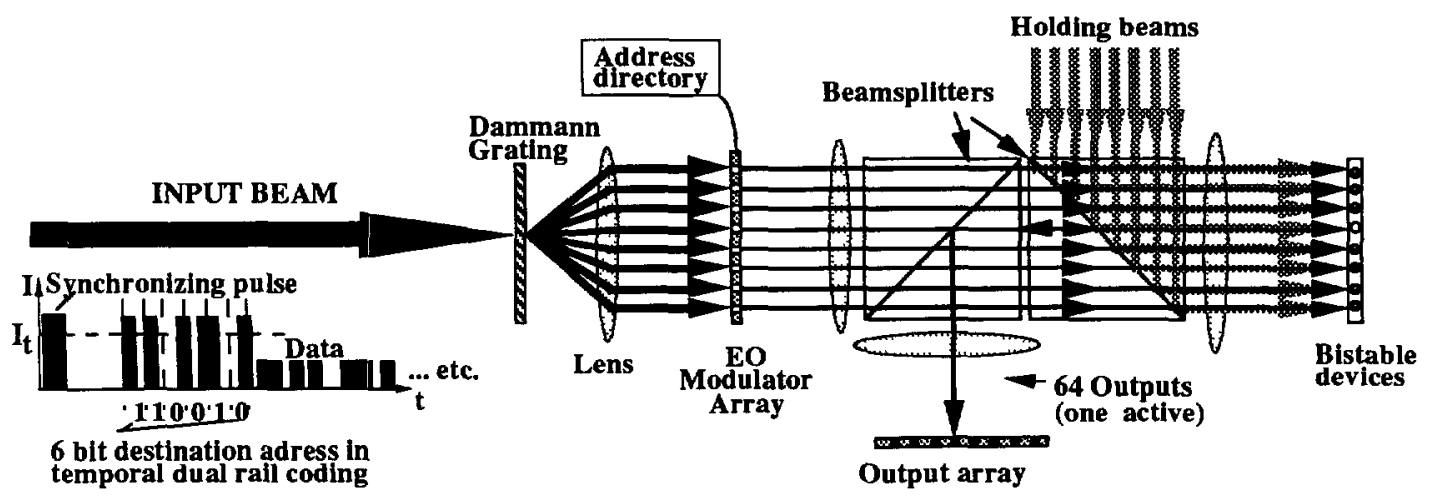

Fig. 1. - Operation scheme (arrows indicate data flow, not real optical beams). 


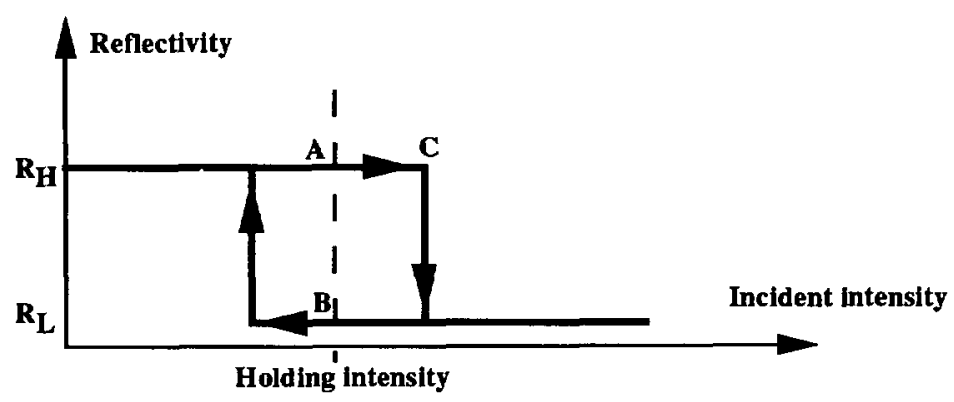

Fig. 2. - Bistability curve.

Signals in the destination channel ( channel address $=110010)$

1

2

3

4

5

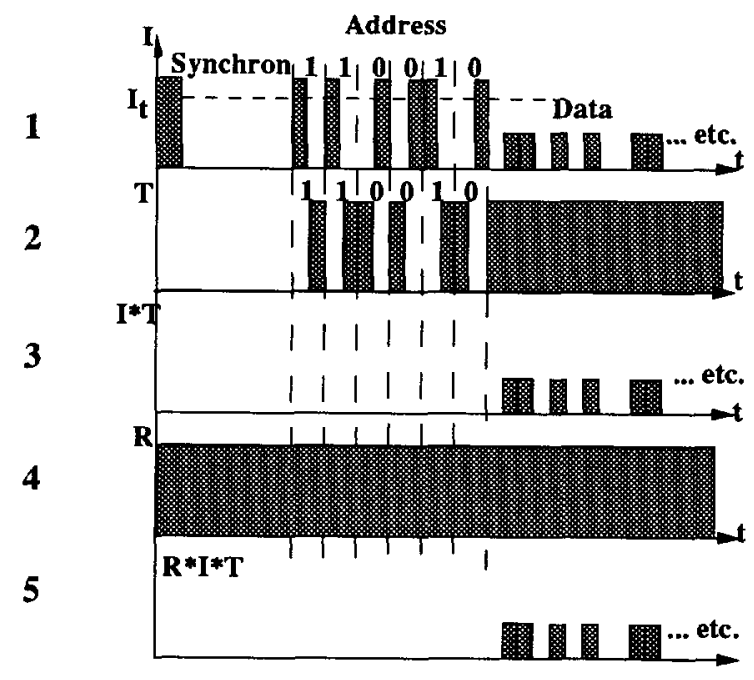

Signals in a non-destination channel ( channel address $=101000$ )

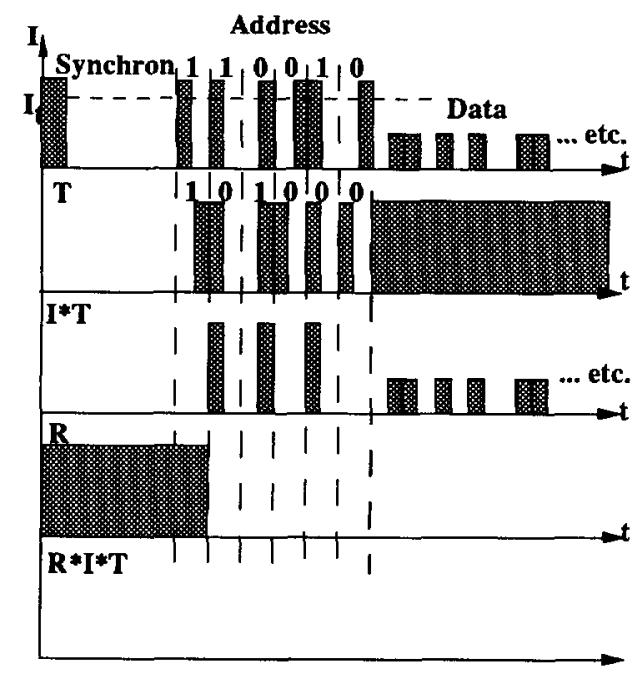

Fig. 3. - Time diagram of the self-routing operation (1) Input signal, 2) Transmission of the modulator element, 3) Signal transmitted by the modulator, 4) Reflectivity of the corresponding bistable element, 5) Signal reflected by the bistable $=$ output).

output channel to be selected. The incident signal beam is divided into 64 identical beamlets using an array illuminator. Each of these sub-beams is focused onto one elementary modulator of the 8 by 8 array. By this fan-out operation, all modulator elements receive the same signal at the same instant. The synchronising pulse of the arriving packet triggers each modulator pixel to deliver its own address in inverse dual rail coding (high-low sequence is for bit 0 , low-high for bit 1). This modulation must be in perfect synchronism with the arrival of the destination address on the modulator array (see row 2 of Fig. 3). The emerging intensity from each modulator element is the bitwise product of two sequences, the dual rail coded destination address and the inversely coded address of the channel in question (see row 3 of Fig. 3). If and only if the two sequences are complementary, which is equivalent to the identity of the two addresses, there is no high level light pulse passing through the modulator pixel at any time during address emission. Therefore, the selected channel never carries optical intensities that 
exceed the bistable switching threshold defined by point $\mathrm{C}$ in figure 1 . All other modulator pixels transmit a number of high intensity address bits, corresponding to the Hamming distance between the channel address and the destination address. Since the modulator array is imaged onto the bistable cavity, each bistable element receives light emanating from the corresponding modulator pixel. Consequently, the bistable device of the selected channel remains in the high reflectivity state, while all other devices switch down to the low reflectivity state (point $B$ in Fig. 2). The commutation is triggered by the first pulse exceeding the threshold (see row 4 of Fig. 3). The data, coded in intensity levels lower than the switching threshold, are thus reflected only by the selected bistable element. During data flow all elementary modulators are set to the transmitting state, while bistable devices remain unchanged and route the entire data string to the selected output port. Before the arrival of the next packet, all bistable devices are reset to point $\mathrm{A}$ by applying lower holding beam intensities for a short time. This operation generates a dead time of the order of one bit-period.

\section{Experimental work.}

3.1 ACTIVE DEviCES. - To implement the required parallel modulation and the threshold switching operations we investigate the use of active devices based on GaAs/GalAlAs multiple quantum well structures [7]. The first of these devices is an $8 \times 8$ array of transmission type electro-optical modulators [8], which uses the absorption variations induced by an electric field applied perpendicularly to the layered structure. The elementary modulators are individually addressable by the means of a $6 \mathrm{~V}$ electronic control signal added to a $9 \mathrm{~V}$ continuous polarisation. The response time of the modulators is of the order of $1 \mathrm{~ns}$ and the modulation contrast ratio is approximately 2 .

The second active element, the optical bistable device $[9,10]$ consists of a non-linear FabryPérot etalon. The non linearity is provided by the MQW material and the cavity is formed by two Bragg reflectors. In order to obtain an $8 \times 8$ array of bistable devices each element is defined by a separate light spot incident on the same cavity. When maintained in the bistable region of operation by a holding beam array, the devices exhibit a two state memory effect for at least $1 \mathrm{~ms}$. The optical bistable device used in the experiment presents a bistability threshold of $3 \mathrm{~mW}$ and a high to low contrast ratio of 2 .

3.2 EXPERIMENTAL SET-UP. - The optical set-up of the demonstrator shown in figure 4 involves three basic parts : the emission, the routing and the reception units. The task of the emission unit is to generate the input signal by amplitude modulating the continuous beam from a $\mathrm{Ti}$ : saphir laser. To accomplish this task, we use a computer controlled acousto-optical modulator. The routing unit described previously includes the active devices, the imaging optics, two fan-out elements (Dammann gratings [11] designed and fabricated at Institut d'Optique and CNET) generating the signal and holding beams, an other acousto-optical modulator performing the holding beam control (erasure of the bistable) and the polarisation components used to combine the signal and holding beams and to separate the outputs (signal beams reflected by the bistable devices). The reference addresses are introduced in the modulator array by means of an electronic circuit triggered by the synchronisation pulse preceding each packet. The reception unit will comprise 64 receivers connected to a computer in order to verify routing and establish bit error rates. The emission and reception modules are an integral part of the present set-up. Moreover, they can in principle be placed a long distance from the routing unit, connected by a single optical fibre transmitting data packets and control bits.

3.3 DATA ROUTING EXPERIMENTS. - For the demonstration of address decoding in one channel of the system, the set-up of figure 4 was slightly modified. The two Dammann 


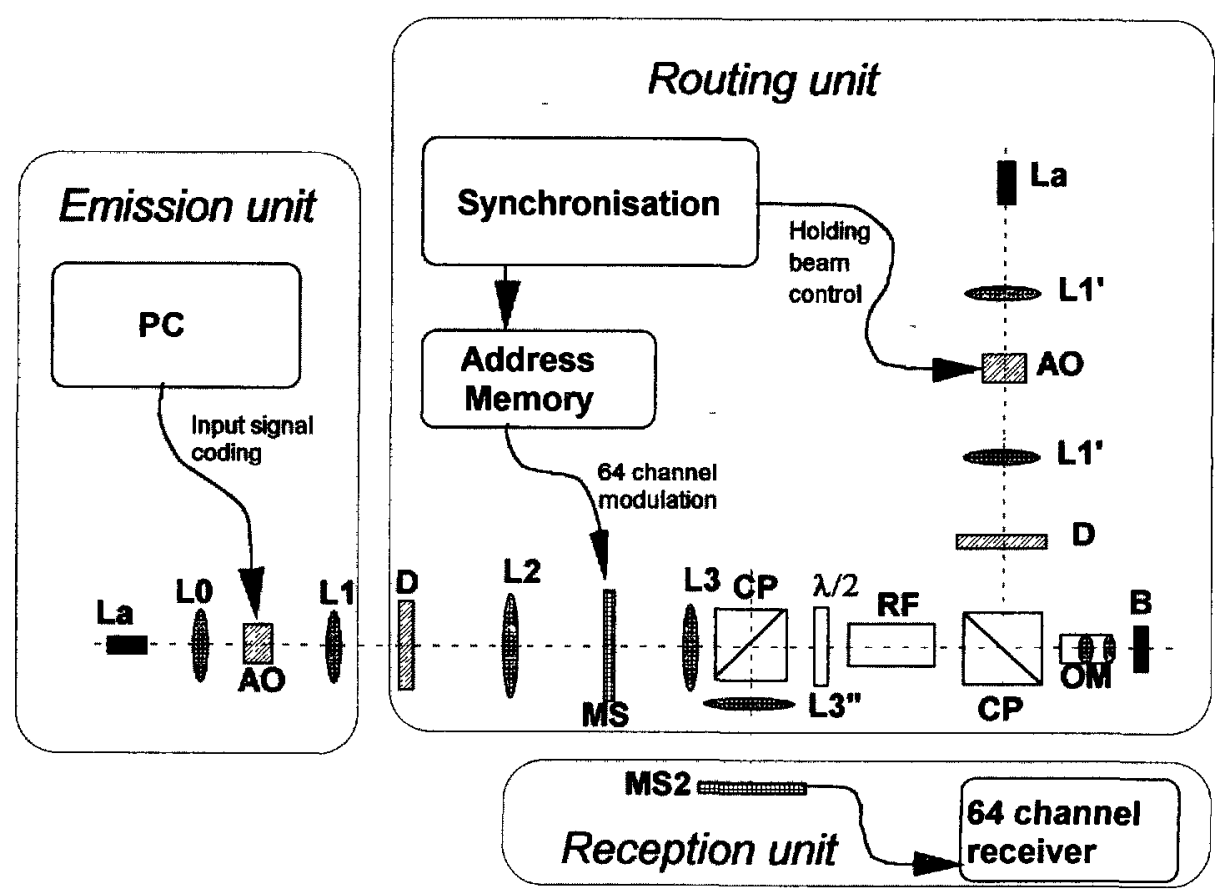

Fig. 4. - Optical set-up of the demonstrator system (Notations : La : Laser, AO : Acousto-optical modulator, OM : microscope objective, L : Lens, D : Dammann Grating, MS : MQW electro-optical modulator array, RF : Faraday Rotator (facultative), L/2 : Half wave plate, $\mathrm{Cp}:$ Polarising beamsplitter. B : MQW optical bistable device, PD : Photodetector array).

gratings were removed in order to illuminate a single pixel of the spatial light modulator and one point on the bistable cavity. The detector array was replaced by a single receiver. The address of the observed channel was 0 (010101010101 in temporal dual rail coding). Figure 5 shows the response of the system to four different input signals. The destination address of the first packet matches the reference address of the observed channel, thus all 6 high level address bits are attenuated by the modulator pixel. The intensity of these pulses added to the holding beam does not exceed the switch-off threshold of the bistable device, so it remains in the high reflectivity state. The synchronising pulse does not initiate the commutation because it arrives before the holding beam is on. If the two addresses do not match exactly, the first high level address bit transmitted by the modulator commutes the bistable device into the low reflectivity state. This case is illustrated in figure 5 by the three other oscilloscope traces corresponding to three different destination addresses. The non-zero intensity of these blocked packets is due to the bistability contrast ratio of two. Considerably higher contrast ratios have been achieved with other samples [12], but their use with the actual spatial light modulators is not possible because of wavelength incompatibility (the SLM works at $864 \mathrm{~nm}$ as well as the bistable device used in the experiment, while $\lambda=838 \mathrm{~nm}$ for the high contrast sample).

In this experiment the system operated with 10 byte long data packets at $3.5 \mathrm{~mW}$ incident power on the bistable ( $3 \mathrm{~mW}$ holding beam plus $0.5 \mathrm{~mW}$ signal beam). A more stable operation was observed at slightly higher intensities (about $10 \mathrm{~mW}$ ) allowing the operation with packet lengths up to 1 kbyte. The clock frequency of the system is fixed at $20 \mathrm{MHz}$ due to our experimental means and not related to device response times being of the order of ns. The operation of the system at address bit periods considerably shorter than $50 \mathrm{~ns}$ would require 


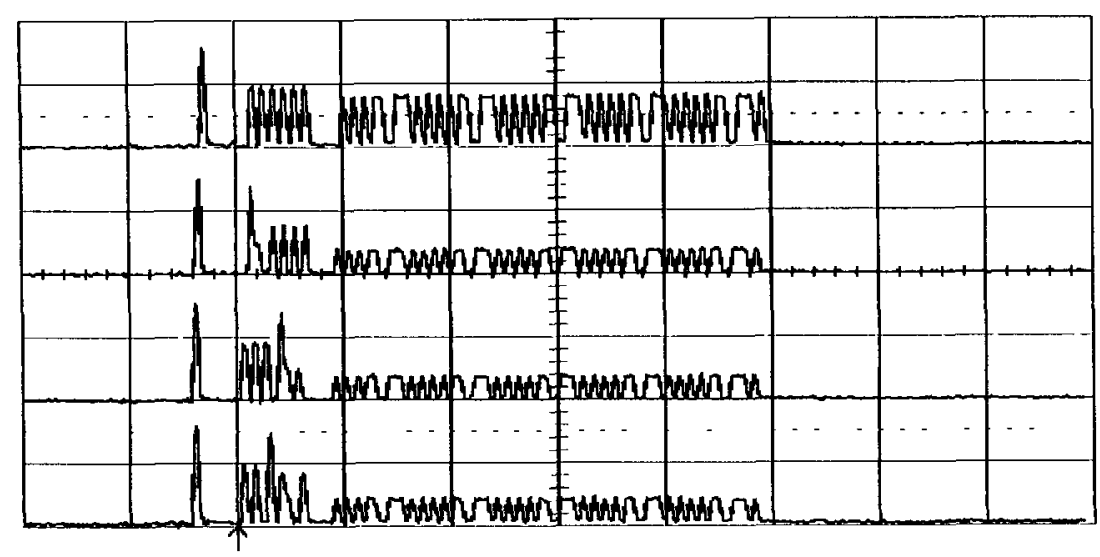

Fig. 5. - Output signals of one channel of the switch. The first oscilloscope trace shows a packet with a destination address matching the address of the observed output channel, so the packet is transmitted by the system. The three other traces carry addresses pointing to an other output, resulting in an attenuated signal in the observed channel.

slightly higher intensity levels. The data bit rate can be increased independently of device limitations because the system is entirely transparent to data.

To estimate the robustness of the address decoding an experiment with automatic emission and reception was performed. A series of packets was generated carrying randomly chosen addresses with a probability weight of 0.5 on the address of the observed channel. The output of the system was recorded by a digital oscilloscope. A sample series of 100 packets were recorded without any errors in the address decoding. However, for $15 \%$ of the packets the duration of the memory effect was not sufficiently long since a parasitic commutation occurred before the end of the data sequence. This problem is essentially due to amplitude fluctuations of the input laser beam. Error free operation could be maintained only for some minutes without tuning, probably because of thermal drifts. Further efforts will be made to reduce noise and thermal variations below $1 \%$ in order to demonstrate a more stable operation of the system.

\section{Conclusions.}

First experimental results on an optical packet switching system were reported. The routing principle based on the optical decoding of header addresses was demonstrated by one channel operation of the system. Further experiments will be carried out to perform error free and parallel operation of the demonstrator. A longer term objective is to prove the feasability and the efficiency of the described self-routing principle in crossbar or multistage interconnection networks and demonstrate the miniaturisation of bistable device based switching systems.

\section{Acknowledgments.}

This work is supported by the French Ministry of Research under the project MOTS/OSTI 91.

\section{References}

[1] Lalanne Ph., Chavel P., Perspectives for Parallel Optical Interconnects, Esprit Basic Research Series (Springer, 1993). 
[2] McCormick F. B. et al., Six-stage digital free-space optical switching network using symmetric self-electro-optic-effect devices, Appl. Opt. 32 (1993) 5153-5171.

[3] Sawchuk A. A., Jenkins B. K., Raghavendra C. S., Varma A., «Optical interconnection networks », Proc. 1985 International Conference on Parallel Processing (August 1985) pp. 388-392.

[4] Fracès M. et al., « Multiprocessor based on an optical crossbar network », Proceedings of Optical Computing'88, SPIE, Vol. 963, Toulon (1988) p. 36.

[5] Cloonan T. J., McCormick F. B., Lentine A. L., Analysis of control subsystems for free-space photonic switching architectures, Appl. Opt. 31 (1992) 2587.

[6] Buller et al., All-optical routing networks based on bistable interferometers, Appl. Phys. Lett. 53 (1988) 2465.

[7] Pocholle J. P., Propriétés optiques des matériaux semiconducteurs à puits quantiques et applications dans le domaine du traitement du signal, l'Ecole d'été d'Optoélectronique, Cargèse, 1989 (Les Editions de Physique, 1990).

[8] Schnell J. Ph., Raffy J., Pocholle J. P., Delboulbe A., Dutrey G., Lehoux J., Werner M., Papuchon M., Huignard J. P., Two-dimensional multiquantum well spatial light modulators for interconnections and optical processing, Technical Digest of Spatial Light Modulators, Vol. 14 (1990) pp. 60-64.

[9] Oudar J. L., Kuszelewicz R., Sfez B., Pellat D., Azoulay R., Quantum well nonlinear microcavities, Superlattices and Microstructures 12 (1992) 89.

[10] Rivera T. et al., Reduced threshold all-optical bistability in etched quantum well microresonators, Appl. Phys. Lett. 64 (1994) 869.

[11] Jahns J., Downs M. M., Prise M. E., Streibl N., Walker S. J., Dammann gratings for laser beam shaping, Opt. Eng. 28 No. 12 (1989).

[12] Sfez B. G., Oudar J. L., Michel J. C., Kuszelewicz R., Azoulay R., "High contrast multiple quantum well optical bistable device with integrated Bragg reflectors, Proc. Conf. Nonlinear Dynamics in Optical Systems, Afton (1990). 